\title{
New Probing Technology Now Enables Impedance Controlled On-Wafer Probing
}

\author{
M. Wollitzer ${ }^{1)}$, S. Thies ${ }^{1)}$, S. Schott ${ }^{2)}$ \\ 1) Rosenberger Hochfrequenztechnik, Hauptstrasse 1, D-83413 Fridolfing, Germany \\ M Wollitzer@Rosenberger.de / S Thies@Rosenberger.de
}

2) Karl Suss Dresden GmbH, Suss-Strasse 1, D-01561 Sacka, Germany sschott@sussdd.de

\begin{abstract}
The development of coaxial and planar RF measurement systems demands a completely different approach as currently used in the low frequency field. In order to achieve minimum reflections in an RF cable, a defined wave resistance must be kept up along its whole length. It is therefore of primary importance to choose appropriate manufacturing techniques, in this case micromachining, after a thorough analysis of all possibilities for HF transmission. We will show that applying these principles to the tips used to contact planar circuits results in the smallest possible impairment to the signals to be transferred.
\end{abstract}

\section{INTRODUCTION}

Wave conductors insulated by air as dielectric show the smallest possible attenuation. Using dielectrics with a higher dielectric constant (always necessary to define the position of the conductors) decreases propagation speed. In this case the dimensions of the conductors have to be scaled down inversely proportional to the dielectric constant to achieve similar electrical behaviour. Highest precision is demanded during development and manufacturing of HF cables and connectors to ensure smooth, seamless and thus low reflection transitions.

Reflective loss can, for example, be kept to less than $10 \mathrm{mU}$ at up to $40 \mathrm{GHz}(1 \mathrm{mU}=$ $1 / 1000$ of signal voltage) in a common $2.92 \mathrm{~mm}$ precision connector.

The development of the SUSS-ROSENBERGER /Z/-Probe is the first success in manufacturing a probe tip up to $40 \mathrm{GHz}$ which has internal reflections not significantly higher than those of the cable connectors. An exceptional detail is that this RF probe only uses air to insulate the RF conductors. It therefore shows exceptionally low reflections and insertion loss. They have furthermore proved to have a high power handling capability and low intermodulation.

\section{/Z/-PROBE CONCEPT}

At the core of the /Z/-Probe is an air-dielectric coplanar line as the contacting device, which is formed by conductors with a rectangular section. A controlled impedance is defined by the ratio of width to height of the air gap between the contact springs. Even in the area where the springs are held in a dielectric, the gap can be designed to achieve the same impedance. A schematic view of the contact spring assembly and the transition to the coaxial air line is shown in diagram 1. The central conductor of the coax line ends directly on the inner strip of the coplanar wave-guide. Depending on the frequency range, the /Z/-Probes are available with $2.92,2.4$ or $1.85 \mathrm{~mm}$ coaxial connectors. 
Diagram 2 is a time domain plot of the reflections in a /Z/-Probe. The transition from the connector to the probe is shown between the two markers "1", followed by the transition into a $50 \Omega$ calibration load. Further the reduction of the electrical length due to the /Z/-Probe's air isolation compared to a coaxial cable probe with its high dielectric constant can be observed (markers 1-2). It is clearly to be seen that reflection peaks have been reduced to about half the amount of a standard probe.

\section{IZI Probe Design $\quad$ Air isolated}

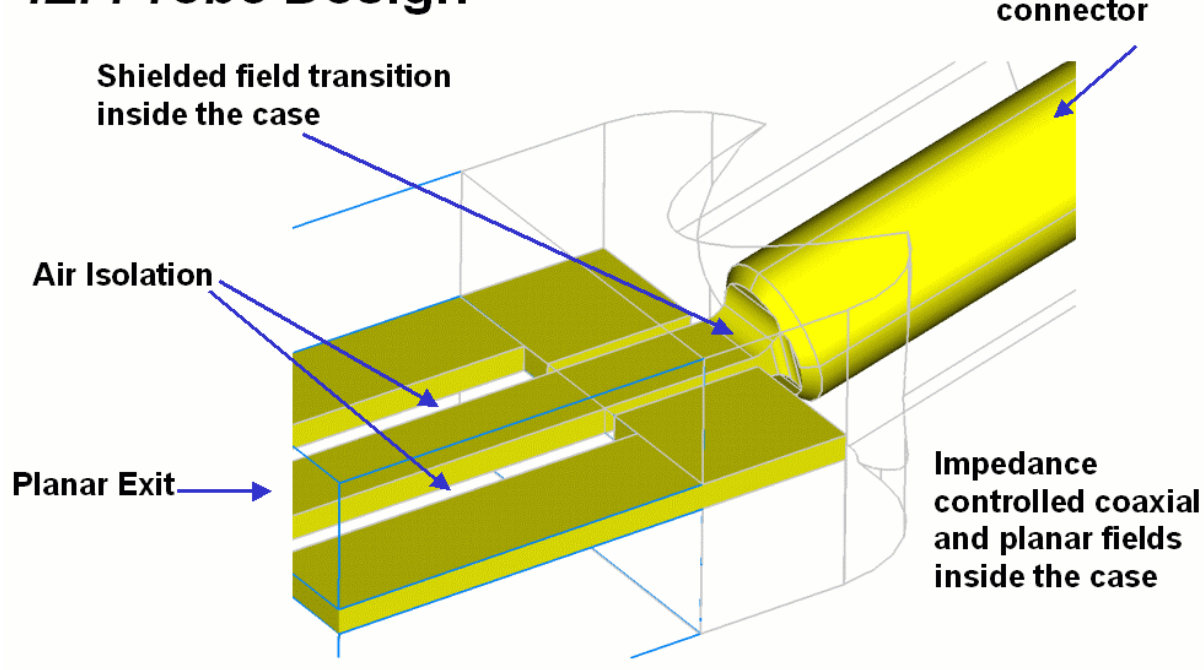

Diagram 1: Internal structure of the /Z/-Probe
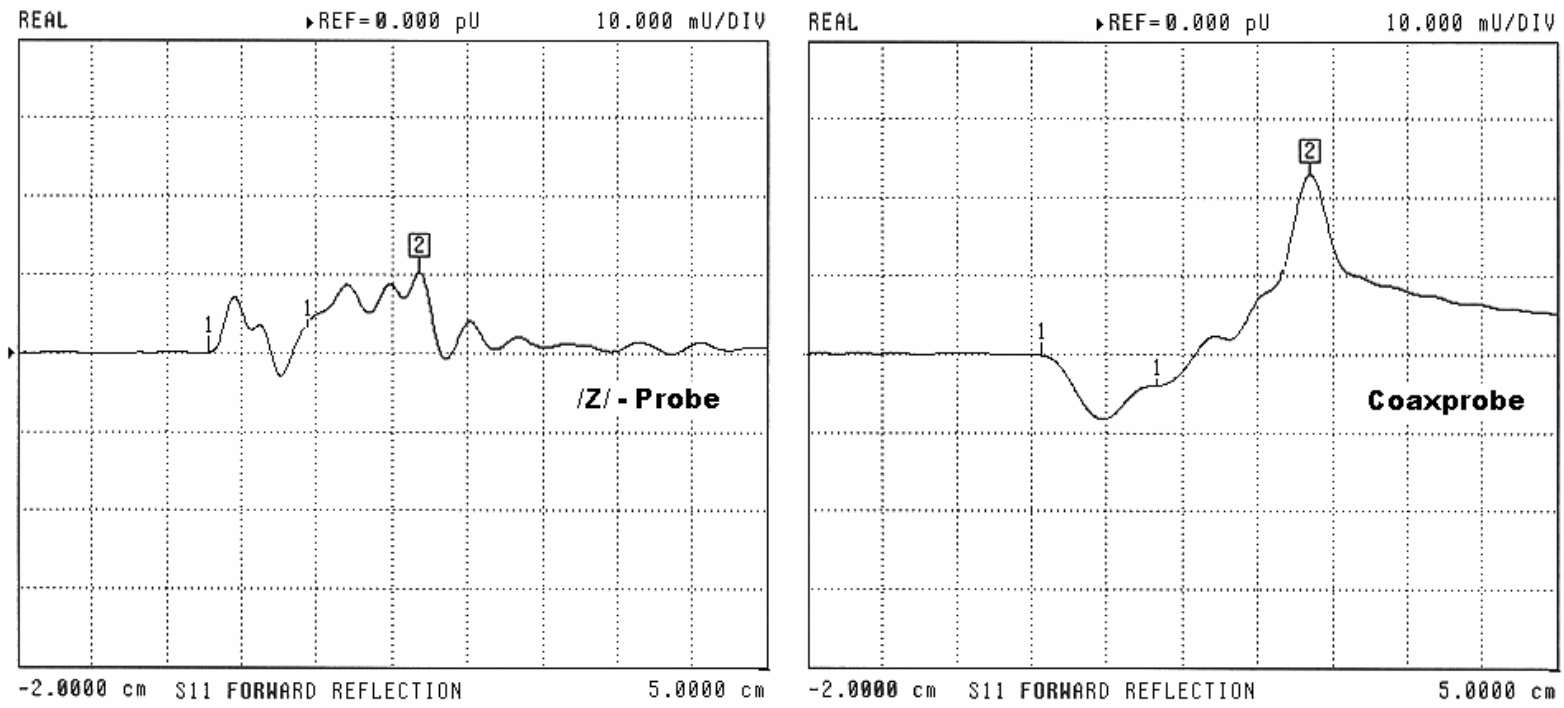

Diagram 2: Time-Domain Plot of the /Z/-Probe (left), Right: results from technology used previously 
The planar structures are manufactured using the highly precise UV-LIGA technology. $100 \mu \mathrm{m}$ was decided upon as the thickness for the planar structures. An impedance of $50 \mathrm{Ohms}$ is then achieved by a gap of approx. 60 microns between each conductor of the coplanar line. In order to ensure this, the thickness of the springs and the distance between them has to be kept within a tolerance of about 1 micron (see Fig. 3). The precision of the UV-LIGA is kept during assembly because the three planar components are fixed onto a dielectric support before separating them. The front ends form the contact springs, which can move independently from each other due to the air gaps.
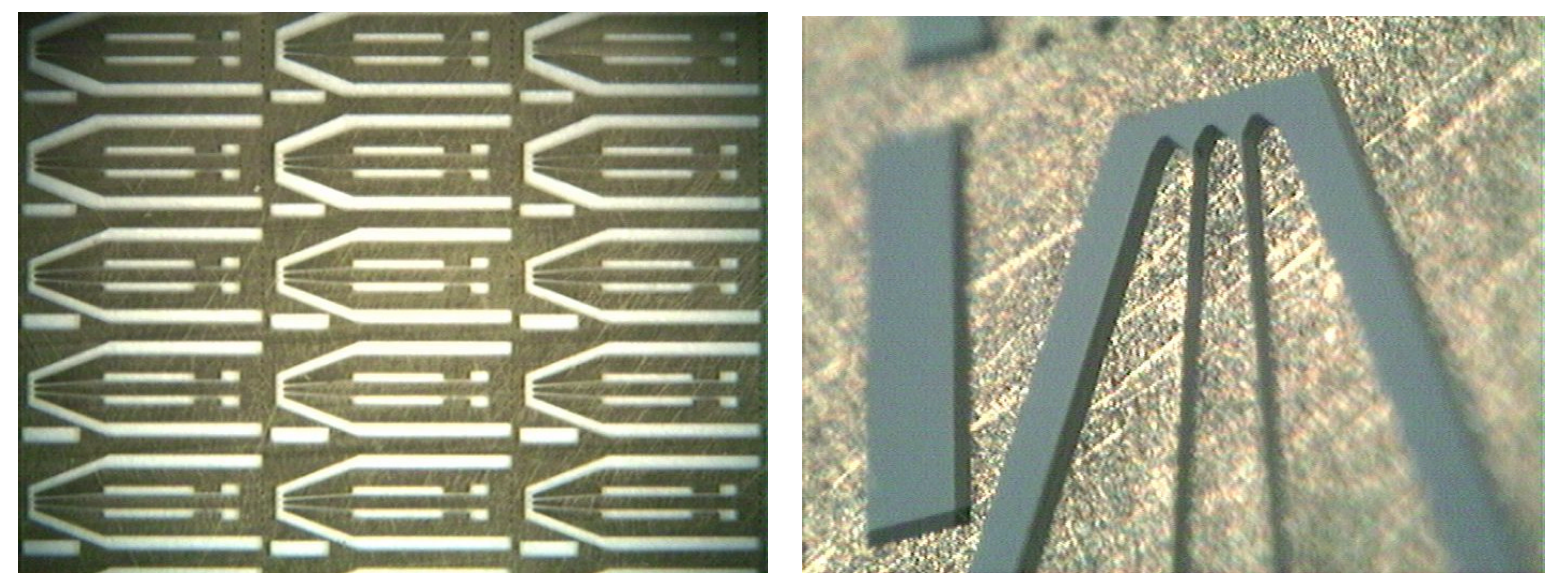

Pictures 3 a \& b: UV-LIGA Spring Contact Structures

Only recent developments in UV-LIGA technology have made the design of this new probe tip possible. New technologies have been developed which enable a highly accurate parallelism of the air gap. Currently nickel is used for the tips as it has excellent mechanical properties and is wear resistant.

This was proved by 1.2 million touchdowns on an aluminium-coated wafer. A tester with a high precision piezo actuated $z$ axis was used for this test. A piezo sensor allows measurement of contact force versus $u$ travel. DC resistance was measured after every 10.000 touchdowns. After the initial contact with the wafer, the springs were pushed down further until a rather high contact pressure of $0.3 \mathrm{mN}$ was reached by an overtravel of 50 microns. The wear of the tip was checked using a SEM Microscope.

As can be seen in picture 4, there is very little wear on the tip even after 1.2 million contacts. Clearly to be seen are wear particles of the wafer, which can be removed by any common cleaning process. The tests show that one can expect a much longer life from these tips as the specified 1 million touchdowns. A high contact pressure with roughly $70 \mu \mathrm{m}$ overtravel as is usual with coaxial probes is not necessary with the /Z/-Probe. Tests on planar calibration substrates showed that the amount of overtravel does not have an effect on the measurement result. This ensures a repeatability superior to other approaches. Picture $5 a$ clearly shows the point at which the safe contact of the wafer is reached. After this point DC and RF characteristics stay constant. 
Due to micromachining, the alignment of the /Z/-Probe is clearly better. After assembly, the tips are exactly level. Together with a highly precise SUSS Probe System, levelling of the tips to the surface of the wafer is no longer required. The vertical and horizontal adjustment difficulties experienced with coaxial probes are not experienced with this new Probe.

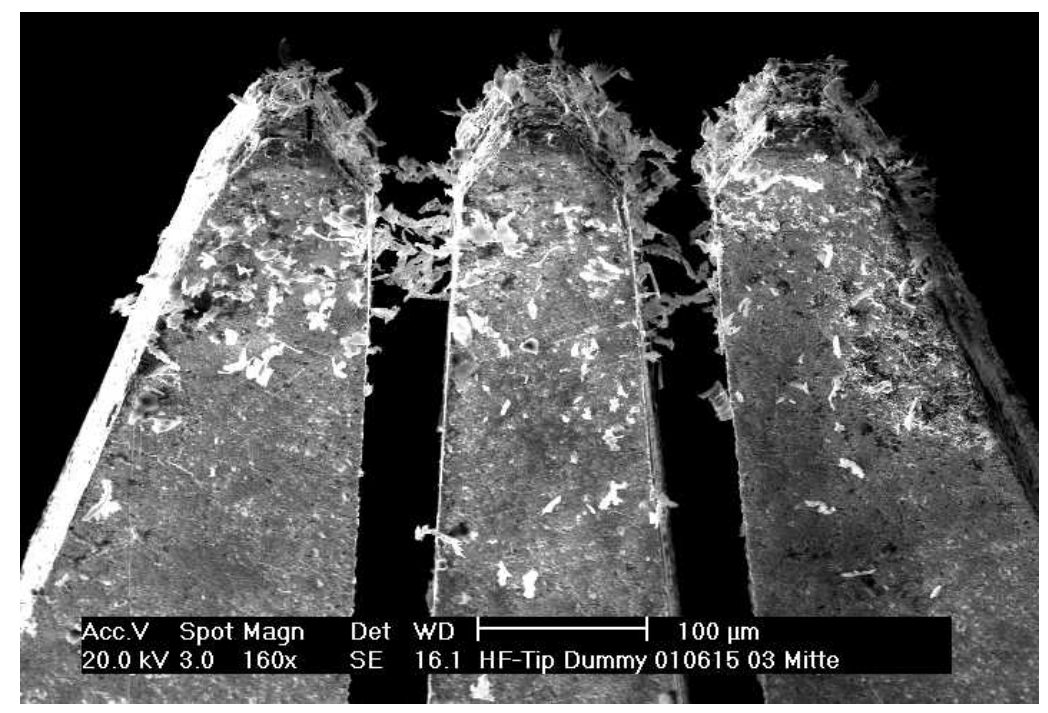

Picture 4: REM Picture of the /Z/-Probe after 1.2 Mio. touchdowns on Al pads
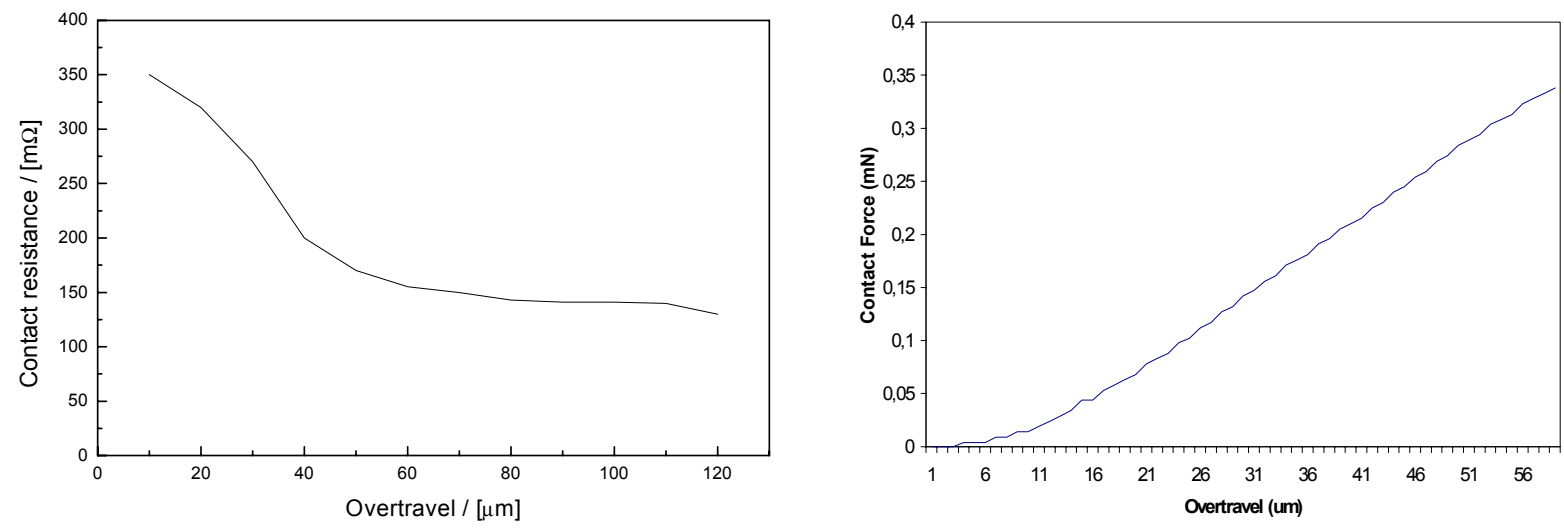

Diagram 5 a \& b: Contact resistance and force on Al test pads

\section{CONCLUSION}

The new /Z/-Probe features not only improved electrical performance, but considerable ease of handling. Levelling is no longer required and precise in-line adjusted contact springs allow reduced overtravel. Further, contacting is very reliable due to comparably long, individually flexing tips, which ensure excellent stability of calibration.

Finally, the /Z/-Probe has a very rugged design. It is therefore virtually impossible to destroy it during normal use. 